\title{
Computational mechano-chemo-biology: a tool for the design of tissue scaffolds
}

\author{
Jose Manuel Garcia Aznar ${ }^{1}$ - Clara Valero ${ }^{1} \cdot$ Carlos Borau $^{1} \cdot$ Noelia Garijo $^{1}$
}

Received: 11 December 2015/Accepted: 21 October 2016/Published online: 5 November 2016

(C) Springer International Publishing Switzerland 2016

\begin{abstract}
Computational modeling is usually employed for simulation, design and manufacturing of tissue scaffolds, specially focused on macroscopic and microscopic properties, relying on anatomy and geometrical constraints. However, these models typically require to take into account the effects of cell-matrix interaction due to its crucial influence on a range of cellular processes including cell adhesion, differentiation and tissue formation among others. Computational mechano-chemo-biology is a numerical approach that aims to consider this interaction by means of a multiphysics and/or a multiscale computer framework. This article reviews some of the recent progress made in modeling bone regeneration induced by scaffolds, taking into account cell-matrix interactions. The issues covered in this work include different kind of numerical models at different length scales that go from cell-matrix interaction to tissue mechanics. The review concludes summarizing the main challenges that researchers face to consolidate modeling as a final design tool for tissue engineering.
\end{abstract}

Keywords Computer-aided tissue engineering . Computational mechano-biology $\cdot$ Cell-material interaction $\cdot$ Scaffold manufacturing

Jose Manuel Garcia Aznar

jmgaraz@unizar.es

1 Aragón Institute of Engineering Research (I3A),

Department of Mechanical Engineering,

University of Zaragoza, Zaragoza, Spain

\section{Introduction}

Currently, computational modeling is one of the tools that is being recognized as an important partner of experimental work in biology [51] and biomedical engineering sciences in different applications like cancer [19], cardiovascular [89], bone [21] and wound healing [86].

One of the computing technologies that is widely used in biomedical applications is CAD (Computer Aided Design), ranging from construction of patient-specific models to customized implant design [58]. 3D anatomy based on computed tomography (CT) or magnetic resonance imaging (MRI) data provides the required technology to create specific models in cardiovascular [31, 39, 83], bone [44] or other applications [61]. For example, Bah et al. [3] recently developed a framework to model the geometric variability of the anatomic structures within a large population of femurs. Vahdati et al. [66] combined gait analysis and a subject-specific musculoskeletal model with subjectspecific bone geometry in a computational bone remodeling methodology to predict bone density distribution. González-Carbonell et al. [26] used the patient-specific geometry and material properties to study tibial torsion. Dao and Pouletaut [18] created integrated contact models for the simulation of knee replacement implants. Also, Carey et al. [12] created subject-specific FE models of the tibiofemoral joint using dynamic stereoradiography data and kinematic analysis. Therefore, it is clear that CAD is a tool especially adequate for the personalized modeling of anatomies and subject-specific geometries. This circumstance has been possible largely thanks to the developments made in imaging technologies and analysis.

Computer-aided technologies are also especially useful for advanced modeling and simulation in regenerative medicine, where subject-specific characteristics of the 
injury have to be taken into account. Indeed, one particular strategy in regenerative medicine is tissue engineering, where biomaterials are used in combination with cells and other stimuli to promote self-repair of injured tissues. Hence, the design of these biomaterials, normally known as scaffolds, is fundamental to induce an adequate healing. These scaffolds, present an specific 3D architecture that aims to mimic the complex behaviour of extracellular matrix in healthy tissues [81]. Their design is defined by the properties of the biomaterial itself and the architecture characteristics. Recently, tissue engineering (TE) has benefited from the development of additive manufacturing (AM) techniques in combination with computer-aided technologies in a novel field known as Computer-Aided Tissue Engineering (CATE), which have led to the design and fabrication of porous scaffolds with custom-tailored architectures [25, 46, 79]. CATE combines different technologies [81], like computer-aided design (CAD), medical image processing, computer-aided manufacturing (CAM), and solid freeform fabrication (SFF) for three major applications in tissue engineering:

1. computer-aided tissue modeling, including 3D anatomic visualization, 3D reconstruction, CAD-based tissue modeling, and bio-physical modeling for surgical planning and simulation;

2 tissue scaffold modeling and biomimetic design, including computer-aided scaffold design and application for virtual scaffold characterization, biomimetic design under multi-constraints, and multi-scale modeling of biological systems incorporating interaction with scaffolds;

3. bio-manufacturing for tissue and organ regeneration, including computer-aided manufacturing of tissue scaffolds, bio-manufacturing of tissue constructs, bioblueprint modeling for 3D cell and organ printing.

One of the applications where CATE is being successfully applied is Bone Tissue Engineering [32, 80]. These computer-based works mainly focus on the control and design of the overall shape of the scaffold to match patientspecific anatomical constraints and the internal architecture of the scaffold (pore size, porosity, permeability). Therefore, a heterogeneous scaffold can be designed according to the specifications of each patient and of their injuries. However, these computational works are mainly based on the most adequate scaffold design and preoperative plans, but do not analyze the impact of one specific design on the biological response of cells at the cellular level. As the structural and functional unit of life, cells actively sense the surrounding mechano-chemical microenvironment and respond accordingly to regulate tissue formation and healing $[9,15,54,85]$. Actually, the cell-scaffold interplay exerts an essential role during bone regeneration as cells adhere to the scaffold surface, differentiate and secrete new tissue (see Fig. 1). Therefore, it is fundamental to advance in the understanding of how scaffold properties (biomaterial and architecture) may regulate cell response at scaffold level and how it can affect to the macroscopic properties of bone tissue, is fundamental. In fact, to understand how different mechano-chemical conditions may also regulate this cell response is a topic of relevant interest. Both aspects are normally studied by the field of Computational Mechano-Chemo-Biology, being its main purpose to predict the long term response of cells for a specific scaffold design and for specific local mechano-chemical microenvironment. This aspect has not been thoroughly studied by CATE, although it is clearly in the spirit of this technology.

Therefore, this article aims to review some of the recent progress made in modeling cell-scaffold interactions at multiple length scales and involving multiple fields of physics. Although many different and diverse methodologies have been used for computational modelling in Tissue Engineering, our review focuses on the multiphysics and multiscale analysis. Hence, the first section presents the multiphysics modeling approach. Next, we show a review of multiscale approaches in tissue engineering. And finally, a more detailed description of models that aim to simulate cell-scaffold interactions is presented. The review concludes with a look at future opportunities and challenges to be faced in computational modeling of tissue regeneration.

\section{Multiphysics modeling in tissue engineering}

Due to the wide variety of physic fields involved (solid mechanics, fluid flow, mass transfer or biochemistry among others), many different works based on computational

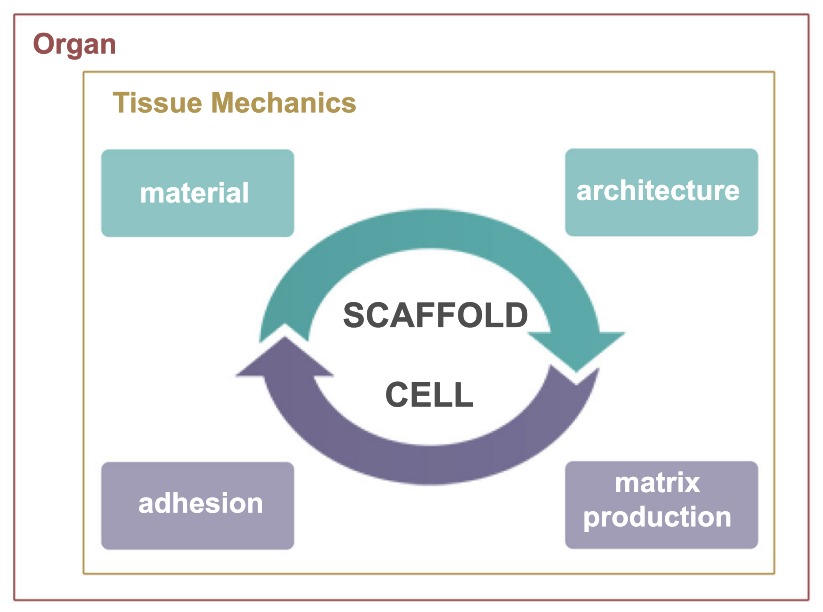

Fig. 1 Interplay between cells and scaffold. When the scaffold is implanted, cells adhere to it to produce matrix. Matrix production is determined by the scaffold properties (material and architecture) which subsequently evolve due to cellular activity 
modeling of tissue engineering phenomena can be found in literature [23]. One possible classification is to divide them into three main categories (see Fig. 2) according to the biophysical stimuli that regulates tissue differentiation and formation: solid mechanics, fluid mechanics and mass transport. Certainly, these stimuli are not used in an isolated way, but as a combination of stimuli that establish regulatory theories in function of these stimuli.

Mechanistic-based models normally establish hypotheses of tissue differentiation and/or tissue formation depending on the solid mechanics variables that describe the macroscopic or microscopic deformation of the scaffolds. Several authors evaluate the strain energy density to quantify bone tissue formation [1,53] like in common bone remodeling theories [17]. For example, Adachi et al. [1] simulated bone tissue regeneration and scaffold degradation within a scaffold unit cell based on a voxel finite element approach, optimizing the scaffold microstructure that provides the desired mechanical function during and after the bone regeneration process. They used the change in total strain energy as fundamental mechanical variable to regulate bone formation. Meanwhile scaffold degradation was due to hydrolysis, which was simply assumed to depend on the water content diffused from the surface to the bulk material, also taking into account the decrease of the mechanical properties of the scaffold. Sanz-Herrera et al. $[68,71]$ also proposed mechanical stimuli to regulate bone formation inside a specific type of scaffold, although they also included cell invasion within the scaffold, which was modeled as a diffusion process based on Fick's law. Bone formation was predicted within idealized or theoretical scaffold geometries and submitted to external mechanical loading. Then, the effects of scaffold material properties such as porosity, stiffness, permeability or degradation rate were analyzed.

However, there exist other biophysics-based theories that hypothesize that fluid shear stress can be the key variable determining cell differentiation in bone [48]. All biological tissues are porous media [36], which means that they contain pores filled with a fluid. These characteristic

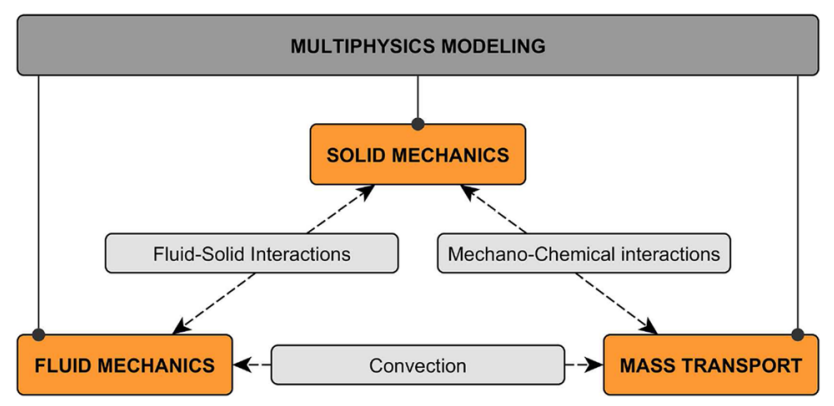

Fig. 2 Classification of multiphysics models according to the involved fields materials produce exceptional mechanical properties to our biological tissues, like impact absorption, lubrication at joints, adequate transport (diffusion and convection) of ions, nutrients and waste products by means of fluid flow, and finally, promoting mechano-sensing mechanims at cell level. Actually, fluid mechanics mainly based on NavierStokes equations, has also been widely used in tissue engineering to estimate wall shear stress magnitudes (WSS) [62], only considering the influence of the scaffold architecture. For instance, several works determined that the distribution of WSS is strongly dependent on scaffold architecture [38, 59].

All these models assume that there exists no interaction between the mechanics of the scaffold and the movement of the fluid within the scaffold pores. However, the mechanical deformation of the scaffold may induce the movement of the fluid inside the scaffold, or equivalently, the fluid flow can generate strains on the scaffold. Therefore, a coupled formulation is required where fluid-solid interaction is considered. Two main numerical approaches have been used to model this phenomenon. The first one is based on the use of fluid-structure interaction (FSI) models, where fluid is simulated by means of Navier-Stokes equations combined with the corresponding equations of solid mechanics and the solid-fluid interface. The second one is known as Darcy's law which describes the flow of a fluid through a porous deformable medium. Actually, the Darcy's law has been derived from the Navier-Stokes equations via homogenization [90].

Fluid-structure interaction (FSI) approaches have been applied to understand the role of scaffold stiffness and architecture on the wall shear stress distribution. In fact, McCoy et al. [50] determined that he applied flow rate dominated the mechanical stimulation when compared to the pore size in collagen-GAG scaffolds. More recently, Zhao et al. [93] also applied this method to investigate the role of scaffold geometry (architecture, pore size and porosity) on pore wall shear stress (WSS) under a range of different loading scenarios (namely: fluid perfusion, mechanical compression and a combination of perfusion and compression), finding that scaffold geometry (spherical and cubical pores), and in particular the pore size, has a significant influence on the stimulation within the scaffolds. In addition, they concluded that the combination of loading conditions would allow amplifying these wall shear stresses.

In addition, fluid-structure interaction has also been used to simulate how the fluid movement deforms the cell body, modeling the cell as a solid in many different applications of tissue engineering. Vaughan et al. [87] developed a fluid-structure interaction model to characterise the deformation of integrin and primary cilia-based mechanosensors in bone cells under fluid flow stimulation. Actually, fluid flow through a channel region of a pressure-driven parallel- 
plate flow chamber was interacting with a single bone cell which was adhered to the bottom wall of the channel region and simulated as a solid. There are many models [47] that investigate the role played by fluid flow on the primary cilia deflection and how this deflection is involved in the mechanotransduction process whereby fluid flow indirectly induces strains on the internal cell components.

Other authors have, on the other hand, focused on understanding how fluid flow can deform cells when they are moving inside the bioreactor, helping to determine the fluid flow conditions in the inside. With this purpose in mind, Ruberg and García-Aznar [64] presented an immersed finite element method that allowed the simulation of solid-fluid interactions specially focused on highly deformable elastic bodies in a Stokes flow environment. The method was based on a global balance equation which combined the solid and fluid momentum balances, the fluid mass balance and, in weak form, the interface conditions. The method resulted in a full coupling of the solid-fluid system which is solved by an exact Newton method. This kind of models are very useful because they allow estimating the stresses and strains that cells are bearing inside the scaffold in different conditions both during cell culture and when this scaffold is implanted.

Nevertheless, different approaches are possible, for instance, considering that our tissues and scaffolds are porous media. In fact, Prendergast and Huiskes et al. [35] presented a biphasic approach based on the Darcy's equation to define a mechanoregulatory phenomenological law, which proposes a combination of solid and fluid mechanics stimulus to define tissue differentiation and formation. They assumed that the relative velocity between fluid and solid and shear strain are the main mechano-fluid stimuli. Indeed, this law has been widely used to simulate bone fracture healing conditions in Finite Element-based models [27, 30] and also to simulate bone tissue formation and cell differentiation [11, 40, 53]. For instance, Kelly and Prendergast [40] determined the influence of scaffold material properties on chondrogenesis in a finite element model of an osteochondral defect, predicting that increasing the stiffness of the scaffold increases the amount of cartilage formation and reduces the amount of fibrous tissue formation in the defect. Byrne et al. [11] developed a fully three-dimensional model for the computer simulation of tissue differentiation and bone regeneration in a regular scaffold as a function of porosity, Young's modulus, and dissolution rate, all of this done under both low and high loading conditions. Milan et al. [53], predicted homogeneous mature bone tissue formation under strain levels of $0.5-1 \%$ at strain rates of $0.0025-0.005 \mathrm{~s}-1$, finding that, under higher levels of strain and strain rates, the scaffold shows heterogeneous mechanical behavior which leads to the formation of a heterogeneous tissue with a mixture of mature bone and fibrous tissue.
More recently, Guyot et al. [29] proposed a combination of numerical methods to solve the fluid-solid interaction problem in a scaffold where bone is growing. They proposed to divide the whole domain of the scaffold in three parts: biomaterial, neotissue and void. Hence, the fluid flow profile is treated differently in each domain using respectively the Brinkman's law, Darcy's law and Stokes, aiming to determine an accurate estimation of the shear stress profile.

The third main category of multiphysics models are based on mass transport by diffusion phenomenon. Actually, there are many macroscopic models that use the diffusion of growth factors as regulatory elements for mediating cellular processes in regenerative process in bone healing [4] and bone regeneration [24]. However, most of these models are not based on pure diffusion transport approaches, but involve convection too, also regulating the transport of these growth factors by means of fluid flow. The production of these growth factors is normally associated to the cells but mediated by the mechanical stimuli that cells are bearing. Therefore, mechano-chemical relations have to be defined. In a recent work, Nava et al. [57] proposed that bone growth was regulated by shear stress and oxygen concentration, but also including the volume occupied by the cell and the tissue growth by using a moving boundary formulation. Therefore, this approach allows not only estimating the fluid shear stress for the initial scaffold geometry, but also predicting the dynamical evolution of the tissue growth within the scaffold, guided by the combination of a fluidbased stimulus and oxygen concentration.

Finally, we should show the application of mechanochemical models to perform simulations in tissue engineering. Despite the wide use of mechano-chemical models for the simulation of bone healing or wound healing $[4,24,37,85]$, there are not many computational works assessing the use of growth factor delivery devices in tissue engineering. Ribeiro et al. [65] recently developed a mechanochemical regulatory model to study the effect of bone morphogenetic protein-2 (BMP-2) on bone regeneration. In particular, they did a comparative study of the impact of different strategies to induce healing to a bone large defect, comparing: natural healing, an empty hydrogel implanted in the defect, and a hydrogel soaked with BMP-2 implanted in the defect. The proposed mechanochemical model successfully predicted the positive effect of BMP-2 on the evolution of healing in large bone defects.

\section{Multiscale modeling in tissue engineering}

Multiscale modeling is a technology inherent to all the biological systems, and Tissue Engineering is not an exception. The overall purpose of this methodology is to 
understand material behaviors from a fundamental perspective taking into account all relevant length and time scales, ranging from the atomic scale to the macroscopic continuum viewpoint. Multiscale modeling has to take into account the fine scale requirements, such as allowing particle flow, and at the same time guarantee the large scale functionality and support. There are many computational multiscale models in the literature that describe different mathematical approaches to recreate biological processes that occur in tissue engineering or tissue regeneration $[13,33,78,82,88]$.

Despite the variety of different methods, in this work we focus on those approaches in which there exists a separation of the length scales, based on the asymptotic homogenization theory (AHT) [34, 72, 84]. Normally, at scaffold or microscopic level, a representative volume element (RVE) is chosen to recreate the geometry of the scaffold and involve most of the cellular processes, however, at macroscopic level, the whole organ is simulated. See Fig. 3 showing one example of how both scales can be linked [69]. The fundamental idea is to decompose each fundamental variable into a macroscopic $\overline{\mathbf{V}}(\mathbf{x})$ and microscopic variable $\mathbf{V}^{\prime}(\mathbf{y})$ :

$\mathbf{V}_{\mathbf{T}}(\mathbf{x}, \mathbf{y})=\overline{\mathbf{V}}(\mathbf{x})+\mathbf{V}^{\prime}(\mathbf{y})$

where $\mathbf{y}$ represents the space description at tissue level and $\mathbf{x}$ at macroscopic level. Actually, $\mathbf{V}^{\prime}(\mathbf{y})$ describes the evolution of the variable at the fine scale considering that their average on the RVE is zero.

In order to connect both scales, homogenization and localization numerical techniques have been widely developed based on different multi-scale approaches $[34,63,84]$ for multiple applications. In particular, in the case of bone tissue engineering, in the work of Sanz-Herrera et al. [69], a solid mechanics problem and a transport problem are solved to simulate bone regeneration.
Therefore, they use this technique in order to homogenize stiffness and diffusion tensor, and they use localization in order to determine locally the cell concentration and strain field. This numerical approach represents a promising strategy for the design and optimization of scaffolds, because it allows to developing a multiphysics approach taking into account solid mechanics, fluid mechanics and transport phenomena. Fundamental scaffold design parameters are considered in the model including porosity, pore size, interconnectivity and mechanical properties. Actually, it is worth noting that there exist multiple evidences linking the bone formation process with the scaffold design parameters: pore size [92], pore shape [43], pore interconnectivity [42] and local curvature [29].

Traditionally, many different models in tissue engineering have been working only at the Scaffold level, in order to understand how different mechano-chemical conditions modify the cell response updating the local architecture of the scaffold [1, 14, 75]. However, these models do not link the evolution of the neotissue bone formation within the local scaffold properties with the macroscopic/ organ scale properties.

Computational multiscale modeling has made possible the design and manufacturing of multiple scaffolds in the last years [77]. In fact, multiscale scaffolds have been successfully incorporated into different biological tissues such as heart valves[16], tympanic membrane [56], or cartilage [45].

\section{Modeling cell-material interactions for tissue engineering applications}

To achieve a controlled and reproducible tissue regeneration under multiple different conditions, it is fundamental to understand cell-material interactions [22, 74]. It is
Fig. 3 Multiscale model of a scaffold (tissue level) implanted into the organ level

\section{Organ-Macro level FEM}

\section{Tissue/Scaffold-Meso level Voxel-FEM}

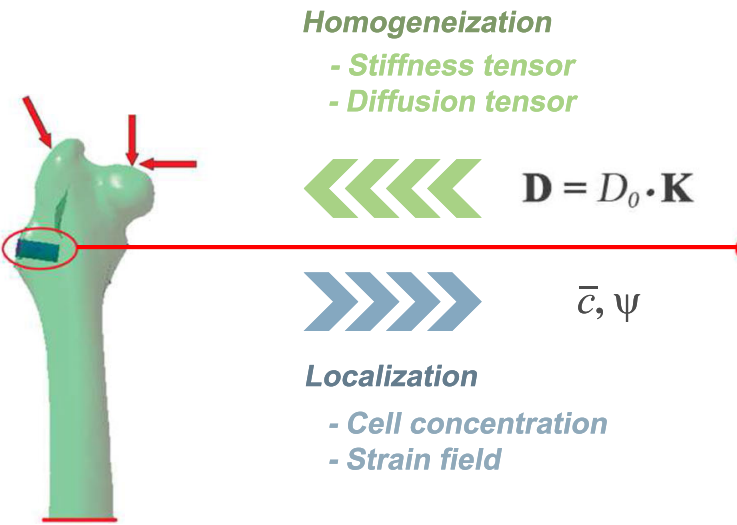

Fully clampled

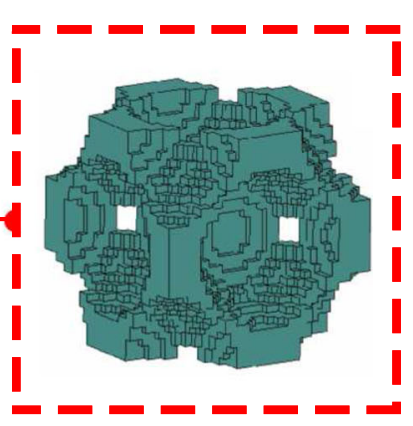


currently accepted that many different kind of biophysical and chemical factors stimulate cells regulating their response. Actually, it is recognized that cells actively sense the mechanical properties of the material in which they are adhered, such as, rigidity, geometry or deformation $[15,54,73]$.

There are many mathematical and computational models in the literature that have focused on modeling the active mechanosensing behaviour in cell-matrix interactions (see [15] for a review). In fact, most of these works have focused on modeling directional cell migration regulated by mechanical properties, such as, durotaxis and tensotaxis [7, 10, 54].

With respect to tissue engineering applications, computational models have been focused on mechanical variables representing the different components of the cell body. For instance, by using a simple model, García-Aznar et al. [22] showed that cell forces increase with stiffness of the material until saturation, orienting the actin stress fibers in this direction. Furthermore, this work also predicts that external mechanical loads may affect cell forces and orientation. The proposed model [8,54] consists on two parallel springs representing the stiffness of the passive mechanical components of the cell and the actin filaments in series with the myosin contractile system (see Fig. 4) . This approach was purely mechanical and static, so that the contractile system exerted a specific force depending on the cell strain, and thus depending on the material stiffness. Additionally, a discrete approach including actin filaments, actin cross-linking proteins (ACPs) and molecular motors, was proposed by Borau et al. [9] to study the mechanosensing phenomenon at the microscopic level. This model evaluated the network contraction and reorganization using different ranges of ECM stiffnesses and actin, ACP and molecular motor concentrations, finding that actin-myosin contractility is one plausible stand-alone mechanism capable of contributing directly to cell mechanosensing.
Topographical cues mimicking the extracellular matrix (ECM), such as curvature, have demonstrated to play a relevant role over a diverse range of cellular behaviours including: initial adhesion, migration, cell growth, differentiation and death [6, 60, 91]. This fact has motivated a high interest in understanding the role of curvature on cell mechanics, thus, several numerical models have been developed to evaluate the stress distribution on the cell body. For example, [22, 73] obtained that substrate curvature determines the stress distribution over the cell, concluding that cell forces are higher in the direction of minimal curvature. Moreover, it showed that stresses become larger at peripheral locations of the cell, since curvature decreases as you move away from the cell centre. This dependence of cell stresses on the scaffold local curvature also helps to understand the phenomenon by which cells tend to align with the direction of minimum principal curvature [76].

Different numerical and theoretical models have postulated the dependence of tissue growth on geometrical features due to the local mechanical forces based on continuum growth theories [5, 20, 67]. More recently, invitro experiments showed that local scaffold topography (curvature) [2, 28, 29, 49, 52] enhances cell growth and neotissue formation. As a consequence, most of these recent numerical models [28, 29] propose phenomenological local growth laws of this neotissue formation, depending on the local mean curvature. From our point of view, this is an indirect way to consider the mechanosensing mechanism that cells use to sense their local mechanical microenvironment.

\section{Future challenges}

There are many challenges that computational mechanochemo-biology needs to face for the optimal design of scaffolds, as recently reviewed in [25]. But, in our opinion,
Fig. 4 Cell-material interaction model of a scaffold (tissue level) implanted into the organ level
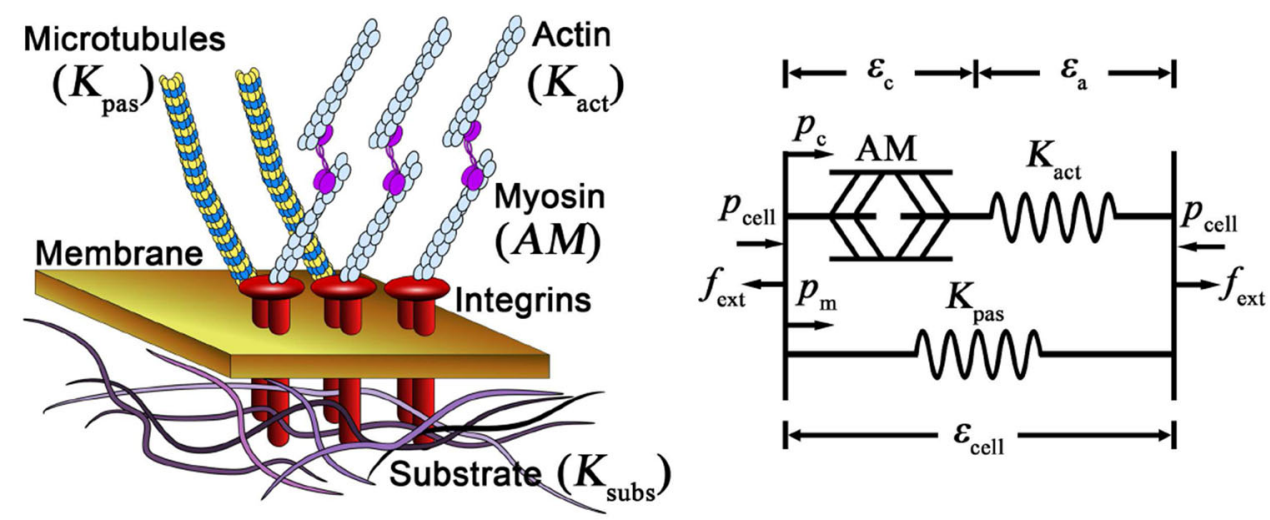
the consideration of the multiple specificities that characterize the tissue that has to be replaced by the scaffold is probably the most crucial aspect. Tissue-specific microenvironment is relevant, but macroscopic patientspecific characteristics, such as geometry, material properties or loads are significant too. In addition, the full integration of the scaffold into the replaced tissue is fundamental, being critical to reduce the relative movement between tissue and scaffold to enhance regeneration at the damaged tissue [55].

To achieve this, it would be desirable to integrate multiple fields (involving mechanical, chemical and biological factors) at different length and time scales that allow linking phenomena that occur at organ, tissue and cell scales.

The coordinated mechanical cell-material interaction (with adequate biochemical conditions) may help to define a local favorable mechano-chemical microenvironment for tissue regeneration, allowing to control the preferential movement of cells, their regulated proliferation and differentiation and the corresponding matrix formation. Therefore, one of the most relevant challenges in tissue engineering is the mechanical design of tissue replacements (scaffolds) with sufficient mechanical integrity to bear loads during tissue regeneration that, at the same time, allows the creation of a local favorable mechanical environment to regulate cell behavior. Moreover, we have to keep in mind that to overcome these challenges, the design of scaffolds presents some relevant constrains that need to be taken into account. Firstly, the porous architecture of the scaffold should allow an adequate mass transport and vascularization in order to facilitate the movement of nutrients and the removal of wastes. Scaffold degradation properties must be precisely adjusted to avoid excessive mechanical forces if the material is removed quickly, or to avoid porosity decrease and reduction of mass transport if the material disappears very slowly [70]. Finally, it is necessary to create scaffolds with non-homogeneous properties and combining different kind of materials, which were called by Giannitelli [25] as hybrid scaffolds. These scaffolds would help to achieve adequate local microstructural properties to activate mechano-sensing cell response, but as well, to keep the macroscopic mechanical role that our organs are playing. Additionally, more steps should be given to achieve the clinical translation of this tissue engineering technology. For this aim, it is essential to improve the robustness of this technology, increasing the repeatability in the results of its clinical application. With this idea in mind, Kerckhofs et al. [41] developed recently an innovative approach for robust screening of scaffolds by means of the combination of microCT characterization with empirical modeling. It is, therefore apparent that, computational models have to be designed in order to facilitate the clinical translation of these scaffolds.

\section{Conclusions}

In this article we have presented some recent modeling works in Tissue Engineering, aiming to show the possibilities that computational modeling offers to scaffold design. It is clear that tissue regeneration by means of Tissue Engineering techniques is a complex process that occurs at different length scales regulated through different mechanical, chemical and biological factors.

As we have shown, computational design of scaffolds is not a novel technique, and has been widely used in CATE $[25,80]$. However, as many authors postulate it is still in an early stage [80]. In the future, CATE should provide the use of multiscale and multiphysics modeling techniques to achieve the integration of the macroscopic design of tissue scaffolds with sufficient mechanical integrity to bear loads during tissue regeneration. At the same time, it should allow the simulation of the local mechano-chemical microenvironment that favors the cell activity to produce new matrix. Therefore, the computational design of scaffolds requires both the optimization of macroscopic properties (to support the tissue- and patient-specific conditions) and microarchitecture (to precisely regulate cell behavior).

Acknowledgements This study was supported by the European Research Council (ERC) through Project ERC-2012-StG 306571 and the Spanish Ministry of Economy and Competitiveness (DPI201564221-C2-1-R).

\section{References}

1. Adachi T, Osako Y, Tanaka M, Hojo M, Hollister SJ (2006) Framework for optimal design of porous scaffold microstructure by computational simulation of bone regeneration. Biomaterials 27(21):3964-3972

2. Van Bael S, Chai YC, Truscello S, Moesen M, Kerckhofs G, Van Oosterwyck H, Schrooten J (2012) The effect of pore geometry on the in vitro biological behavior of human periosteum-derived cells seeded on selective laser-melted Ti6Al4V bone scaffolds. Acta Biomater 8(7):2824-2834

3. Bah MT, Shi J, Browne M, Suchier Y, Lefebvre F, Young P, King L, Dunlop DG, Heller MO (2015) Exploring inter-subject anatomic variability using a population of patient-specific femurs and a statistical shape and intensity model. Med Eng Phys 37(10):995-1007

4. Bailon-Plaza A, Van Der Meulen MC (2001) A mathematical framework to study the effects of growth factor influences on fracture healing. J Theor Biol 212(2):191-209

5. Bidan CM, Kommareddy KP, Rumpler M, Kollmannsberger P, Brchet YJ, Fratzl P, Dunlop JW (2012) How linear tension converts to curvature: geometric control of bone tissue growth. PloS One 7(5):e36336

6. Biton YY, Safran SA (2009) The cellular response to curvatureinduced stress. Phys Biol 6(4):046010

7. Borau C, Kamm RD, García-Aznar JM (2011) Mechano-sensing and cell migration: a 3D model approach. Phys Biol 8(6):066008 
8. Borau C, Kamm RD, García-Aznar JM (2014) A time-dependent phenomenological model for cell mechano-sensing. Biomech Model Mechanobiol 13(2):451-462

9. Borau C, Kim T, Bidone T, García-Aznar JM, Kamm RD (2012) Dynamic mechanisms of cell rigidity sensing: insights from a computational model of actomyosin networks. PLoS One 7(11):e49174

10. Borau C, Polacheck WJ, Kamm RD, Garca-Aznar JM (2014) Probabilistic Voxel-Fe model for single cell motility in 3D. In Silico Cell Tissue Sci 1(1):1-17

11. Byrne DP, Lacroix D, Planell JA, Kelly DJ, Prendergast PJ (2007) Simulation of tissue differentiation in a scaffold as a function of porosity, Young's modulus and dissolution rate: application of mechanobiological models in tissue engineering. Biomaterials 28(36):5544-5554

12. Carey RE, Zheng L, Aiyangar AK, Harner CD, Zhang X (2014) Subject- specific finite element modeling of the tibiofemoral joint based on CT, magnetic resonance imaging and dynamic stereoradiography data in vivo. J Biomech Eng 136(4):041004-041004

13. Carlier A, Geris L, Bentley K, Carmeliet G, Carmeliet P, Van Oosterwyck H (2012) MOSAIC: a multiscale model of osteogenesis and sprouting angiogenesis with lateral inhibition of endothelial cells. PLoS Comput Biol 8(10):e1002724

14. Checa S, Prendergast PJ (2009) A mechanobiological model for tissue differentiation that includes angiogenesis: a lattice-based modeling approach. Ann Biomed Eng 37(1):129-145

15. Chen B, Ji B, Gao H (2015) Modeling active mechanosensing in cell-matrix interactions. Annu Rev Biophys 44(1):1-32

16. Cheung DY, Duan B, Butcher JT (2015) Current progress in tissue engineering of heart valves: multiscale problems, multiscale solutions. Expert Opin Biol Ther 15(8):1155-1172

17. Christen P, Ito K, Ellouz R, Boutroy S, Sornay-Rendu E, Chapurlat RD, van Rietbergen B (2014) Bone remodelling in humans is load-driven but not lazy. Nat Commun 5. doi:10.1038/ ncomms5855

18. Dao TT, Pouletaut P (2015) A hertzian integrated contact model of the total knee replacement implant for the estimation of joint contact forces. J Comput Med Article ID 945379. doi:10.1155/ 2015/945379

19. Deisboeck TS, Wang Z, Macklin P, Cristini V (2011) Multiscale cancer modeling. Annu Rev Biomed Eng 13:127-155

20. Dunlop JWC, Fischer FD, Gamsjger E, Fratzl P (2010) A theoretical model for tissue growth in confined geometries. J Mech Phys Solids 58(8):1073-1087

21. García-Aznar JM, Rueberg T, Doblare M (2005) A bone remodelling model coupling microdamage growth and repair by 3D BMU-activity. Biomech Model Mechanobiol 4(2-3):147-167

22. García-Aznar JM, Sanz-Herrera JA, Moreo P (2011) Cell-material communication: mechanosensing modelling for design in tissue engineering. In: Gefen A (ed) Cellular and biomolecular mechanics and mechanobiology. Studies in mechanobiology, tissue engineering and biomaterials, vol 4. Springer, Berlin, pp 451-462

23. Geris L (2013) Computational modeling in tissue engineering. Springer, Berlin

24. Geris L, Gerisch A, Schugart RC (2010) Mathematical modeling in wound healing, bone regeneration and tissue engineering. Acta Biotheor 58(4):355-367

25. Giannitelli SM, Accoto D, Trombetta M, Rainer A (2014) Current trends in the design of scaffolds for computer-aided tissue engineering. Acta Biomater 10(2):580-594

26. González-Carbonell RA, Ortiz-Prado A, Jacobo-Armendriz VH, Cisneros- Hidalgo YA, Alpzar-Aguirre A (2015) 3D patientspecific model of the tibia from CT for orthopedic use. J Orthop 12(1):11-16
27. González-Torres LA, Gómez-Benito MJ, Doblare M, GarcíaAznar JM (2010) Influence of the frequency of the external mechanical stimulus on bone healing: a computational study. Med Eng Phys 32(4):363-371

28. Guyot Y, Papantoniou I, Chai YC, Van Bael S, Schrooten J, Geris L (2014) A computational model for cell/ECM growth on 3D surfaces using the level set method: a bone tissue engineering case study. Biomech Model Mechanobiol 13(6):1361-1371

29. Guyot Y, Papantoniou I, Luyten FP, Geris L (2016) Coupling curvature-dependent and shear stress-stimulated neotissue growth in dynamic bioreactor cultures: a 3D computational model of a complete scaffold. Biomech Model Mechanobiol 15(1):169-180

30. Gómez-Benito MJ, González-Torres LA, Reina-Romo E, Grasa J, Seral B, García-Aznar JM (2011) Influence of high-frequency cyclical stimulation on the bone fracture-healing process: mathematical and experimental models. Philos Trans R Soc Lond A Math Phys Eng Sci 369(1954):4278-4294

31. Hardman D, Semple SI, Richards JM, Hoskins PR (2013) Comparison of patient-specific inlet boundary conditions in the numerical modelling of blood flow in abdominal aortic aneurysm disease. Int J Numer Method Biomed Eng 29(2):165-178

32. Hollister SJ (2005) Porous scaffold design for tissue engineering. Nat Mater 4(7):518-524

33. Holzhtter HG, Drasdo D, Preusser T, Lippert J, Henney AM (2012) The virtual liver: a multidisciplinary, multilevel challenge for systems biology. Wiley Interdiscip Rev Syst Biol Med 4(3):221-235

34. Hughes TJ, Feijo GR, Mazzei L, Quincy JB (1998) The variational multiscale method: a paradigm for computational mechanics. Comput Methods Appl Mech Eng 166(1):3-24

35. Huiskes R, Van Driel WD, Prendergast PJ, Sballe K (1997) A biomechanical regulatory model for periprosthetic fibrous-tissue differentiation. J Mater Sci Mater Med 8(12):785-788

36. Huyghe JMRJ, Loon VRR, Baaijens FPT (2002) VPM Kemenade and TH Smit. We all are porous media, Swets and Zeitlinger

37. Javierre E, Moreo P, Doblare M, García-Aznar JM (2009) Numerical modeling of a mechano-chemical theory for wound contraction analysis. Int J Solids Struct 46(20):3597-3606

38. Jungreuthmayer C, Jaasma MJ, Al-Munajjed AA, Zanghellini J, Kelly DJ, O-Brien FJ (2009) Deformation simulation of cells seeded on a collagen-GAG scaffold in a flow perfusion bioreactor using a sequential 3D CFD-elastostatics model. Med Eng Phys $31: 420-427$

39. Kayvanpour E, Mansi T, Sedaghat-Hamedani F, Amr A, Neumann D, Georgescu B et al (2015) Towards personalized cardiology: multi-scale modeling of the failing heart. PLoS One 10(7):e0134869. doi:10.1371/journal.pone.0134869

40. Kelly DJ, Prendergast PJ (2006) Prediction of the optimal mechanical properties for a scaffold used in osteochondral defect repair. Tissue Eng 12(9):2509-2519

41. Kerckhofs G, Chai YC, Luyten FP, Geris L (2016) Combining microCT-based characterization with empirical modelling as a robust screening approach for the design of optimized CaP-containing scaffolds for progenitor cell-mediated bone formation. Acta Biomater 35:330-340

42. Kim K, Yeatts A, Dean D, Fisher JP (2010) Stereolithographic bone scaffold design parameters: osteogenic differentiation and signal expression. Tissue Eng Part B Rev 16(5):523-539

43. Knychala J, Bouropoulos N, Catt CJ, Katsamenis OL, Please CP, Sengers BG (2013) Pore geometry regulates early stage human bone marrow cell tissue formation and organisation. Ann Biomed Eng 41(5):917-930

44. Lekadir K, Noble C, Hazrati-Marangalou J, Hoogendoorn C, van Rietbergen B, Taylor ZA, Frangi A (2015) Patient-specific biomechanical modeling of bone strength using statistically- 
derived fabric tensors. Ann Biomed Eng (In press). doi:10.1007/ s10439-015-1432-2

45. Levorson EJ, Raman Sreerekha P, Chennazhi KP, Kasper FK, Nair SV, Mikos AG (2013) Fabrication and characterization of multiscale electrospun scaffolds for cartilage regeneration. Biomed Mater 8(1):014103

46. Liebschner MAK (2012) Computer-aided tissue engineering. Springer Protocols, p 356

47. Lim YC, Cooling MT, Long DS (2015) Computational models of the primary cilium and endothelial mechanotransmission. Biomech Model Mechanobiol 14(3):665-678

48. Liu L, Yu B, Chen J, Tang Z, Zong C, Shen D, Wang J (2012) Different effects of intermittent and continuous fluid shear stresses on osteogenic differentiation of human mesenchymal stem cells. Biomech Model Mechanobiol 11(3-4):391-401

49. Mathur A, Moore SW, Sheetz MP, Hone J (2012) The role of feature curvature in contact guidance. Acta Biomater $8(7): 2595-2601$

50. McCoy RJ, Jungreuthmayer C, O-Brien FJ (2012) Influence of flow rate and scaffold pore size on cell behavior during mechanical stimulation in a flow perfusion bioreactor. Biotechnol Bioeng 109:1583-1594

51. Meier-Schellersheim M, Fraser IDC, Klauschen F (2009) Multiscale modeling for biologists. Wiley Interdiscip. Rev. Syst. Biol. Med. 1(1):4-14

52. Melchels FP, Barradas AM, Van Blitterswijk CA, De Boer J, Feijen J, Grijpma DW (2010) Effects of the architecture of tissue engineering scaffolds on cell seeding and culturing. Acta Biomater 6(11):4208-4217

53. Milan JL, Planell JA, Lacroix D (2010) Simulation of bone tissue formation within a porous scaffold under dynamic compression. Biomech Model Mechanobiol 9(5):583-596

54. Moreo P, García-Aznar JM, Doblar M (2008) Modeling mechanosensing and its effect on the migration and proliferation of adherent cells. Acta Biomater 4(3):613-621

55. Moreo P, Prez MA, García-Aznar JM, Doblare M (2007) Modelling the mechanical behaviour of living bony interfaces. Comput Methods Appl Mech Eng 196(35):3300-3314

56. Mota C, Danti S, D’Alessandro D, Trombi L, Ricci C, Puppi D, Dinucci D, Milazzo M, Stefanini C, Chiellini F, Moroni L, Berrettini S (2015) Multiscale fabrication of biomimetic scaffolds for tympanic membrane tissue engineering. Biofabrication 7(2):025005

57. Nava MM, Raimondi MT, Pietrabissa R (2013) A multiphysics $3 \mathrm{D}$ model of tissue growth under interstitial perfusion in a tissueengineering bioreactor. Biomech Model Mechanobiol 12(6):1169-1179

58. Neal ML, Kerckhoffs R (2010) Current progress in patientspecific modeling. Brief Bioinform 11(1):111-126

59. Olivares AL, Marsal E, Planell JA, Lacroix D (2009) Finite element study of scaffold architecture design and culture conditions for tissue engineering. Biomaterials 30:6142-6149

60. Ozdemir T, Xu LC, Siedlecki C, Brown JL (2013) Substrate curvature sensing through Myosin IIa upregulates early osteogenesis. Integr Biol 5(11):1407-1416

61. Palacio-Torralba J, Jimnéz Aguilar E, Good DW, Hammer S, McNeill SA, Stewart GD, Reuben RL, Chen Y (2016) Patient specific modeling of palpation-based prostate cancer diagnosis: effects of pelvic cavity anatomy and intrabladder pressure. Int $\mathbf{J}$ Numer Methods Biomed Eng 32(1):e02734

62. Patrachari AR, Podichetty JT, Madihally SV (2012) Application of computational fluid dynamics in tissue engineering. J Biosci Bioeng 114(2):123-132

63. Pavliotis GA, Stuart A (2008) Multiscale methods: averaging and homogenization. Springer Science and Business Media
64. Rberg T, Garcia-Aznar JM (2016) Numerical simulation of solid deformation driven by creeping flow using an immersed finite element method. Adv Model Simul Eng Sci 3(1):1

65. Ribeiro FO, Gómez-Benito MJ, Folgado J, Fernandes PR, GarcaAznar JM (2015) In silico mechano-chemical model of bone healing for the regeneration of critical defects: the effect of BMP2. PloS One 10(6): $\mathrm{e} 0127722$

66. Rouhi G (2009) A model for mechanical adaptation of trabecular bone incorporating cellular accommodation and effects of microdamage and disuse. Mech Res Commun 36(3):284-293

67. Rumpler M, Woesz A, Dunlop JW, van Dongen JT, Fratzl P (2008) The effect of geometry on three-dimensional tissue growth. J R Soc Interface 5(27):1173-1180

68. Sanz-Herrera JA, García-Aznar JM, Doblare M (2008) A mathematical model for bone tissue regeneration inside a specific type of scaffold. Biomech Model Mechanobiol 7(5):355-366

69. Sanz-Herrera JA, García-Aznar JM, Doblare M (2008) Micromacro numerical modelling of bone regeneration in tissue engineering. Comput Methods Appl Mech Eng 197(33):3092-3107

70. Sanz-Herrera JA, García-Aznar JM, Doblare M (2009) On scaffold designing for bone regeneration: a computational multiscale approach. Acta Biomater 5(1):219-229

71. Sanz-Herrera JA, García-Aznar JM, Doblare M (2009) A mathematical approach to bone tissue engineering. Philos Trans R Soc Lond A Math Phys Eng Sci 367(1895):2055-2078

72. Sanz-Herrera JA, Kasper C, van Griensven M, García-Aznar JM, Ochoa I, Doblare M (2008) Mechanical and flow characterization of Sponceram carriers: evaluation by homogenization theory and experimental validation. J Biomed Mater Res B Appl Biomater 87(1):42-48

73. Sanz-Herrera JA, Moreo P, García-Aznar JM, Doblare M (2009) On the effect of substrate curvature on cell mechanics. Biomaterials 30(34):6674-6686

74. Sanz-Herrera JA, Reina-Romo E (2011) Cell-biomaterial mechanical interaction in the framework of tissue engineering: insights, computational modeling and perspectives. Int J Mol Sci 12(11):8217-8244

75. Sengers BG, Taylor M, Please CP, Oreffo RO (2007) Computational modelling of cell spreading and tissue regeneration in porous scaffolds. Biomaterials 28(10):1926-1940

76. Smeal RM, Rabbitt R, Biran R, Tresco PA (2005) Substrate curvature influences the direction of nerve outgrowth. Ann Biomed Eng 33(3):376-382

77. Soliman S, Pagliari S, Rinaldi A, Forte G, Fiaccavento R, Pagliari F, Franzese O, Minieri M, Di Nardo P, Licoccia S, Traversa E (2010) Multiscale three-dimensional scaffolds for soft tissue engineering via multimodal electrospinning. Acta Biomater 6(4):1227-1237

78. Stolarska MA, Kim Y, Othmer HG (2009) Multi-scale models of cell and tissue dynamics. Philos Trans R Soc Lond A Math Phys Eng Sci 367(1902):3525-3553

79. Sun W, Darling A, Starly B, Nam J (2004) Computer-aided tissue engineering: overview, scope and challenges. Biotechnol Appl Biochem 39(1):29-47

80. Sun W, Starly B, Darling A, Gomez C (2004) Computer-aided tissue engineering: application to biomimetic modelling and design of tissue scaffolds. Biotechnol Appl Biochem 39(1):49-58

81. Sun W, Starly B, Nam J, Darling A (2005) Bio-CAD modeling and its applications in computer-aided tissue engineering. Comput Aided Design 37(11):1097-1114

82. Swat MH, Thomas GL, Belmonte JM, Shirinifard A, Hmeljak D, Glazier JA (2012) Multi-scale modeling of tissues using CompuCell 3D. Methods Cell Biol 110:325

83. Taylor CA, Figueroa CA (2009) Patient-specific modeling of cardiovascular mechanics. Annu Rev Biomed Eng 11:109-134 
84. Terada K, Ito T, Kikuchi $\mathrm{N}$ (1998) Characterization of the mechanical behaviors of solid-fluid mixture by the homogenization method. Comput Methods Appl Mech Eng 153(3):223-257

85. Valero C, Javierre E, García-Aznar JM, Gómez-Benito MJ (2014) A cell-regulatory mechanism involving feedback between contraction and tissue formation guides wound healing progression. PloS One 9(3):e92774

86. Valero C, Javierre E, García-Aznar JM, Menzel A, GomezBenito MJ (2015) Challenges in the modeling of wound healing mechanisms in soft biological tissues. Ann Biomed Eng 43(7):1654-1665

87. Vaughan TJ, Mullen CA, Verbruggen SW, McNamara LM (2015) Bone cell mechanosensation of fluid flow stimulation: a fluid-structure interaction model characterising the role integrin attachments and primary cilia. Biomech Model Mechanobiol 14(4):703-718

88. Walpole J, Papin JA, Peirce SM (2013) Multiscale computational models of complex biological systems. Annu Rev Biomed Eng $15: 137$
89. Weinberg EJ, Shahmirzadi D, Mofrad MRK (2010) On the multiscale modeling of heart valve biomechanics in health and disease. Biomech Model Mechanobiol 9(4): 373-387

90. Whitaker S (1986) Flow in porous media I: a theoretical derivation of Darcy's law. Transp Porous Media 1:3-25

91. Zadpoor AA (2015) Bone tissue regeneration: the role of scaffold geometry. Biomater Sci 3(2):231-245

92. Zeltinger J, Landeen LK, Alexander HG, Kidd ID, Sibanda B (2001) Development and characterization of tissue-engineered aortic valves. Tissue Eng 7(1):9-22

93. Zhao F, Vaughan TJ, McNamara LM (2016) Quantification of fluid shear stress in bone tissue engineering scaffolds with spherical and cubical pore architectures. Biomech Model Mechanobiol 15(3):561-577 